\title{
Percutaneous transhepatic hemodialysis catheters in chronic hemodialysis patients: technique, functional outcome, and complications from a large population study
}

Osama Abuel Naga Khallaf ${ }^{1 *}$ (D), Karim A. Abd El Tawab ${ }^{1}$, Hazem Ibrahim Korashi ${ }^{1}$, Ghada Samir Ibrahim ${ }^{1}$ and Rasha Samir Mohamed ${ }^{2}$

\begin{abstract}
Background: Chronic renal failure is one of the main health problems in Egypt. Arterio-venous fistulas, grafts, as well as conventional sites for placement of the dialysis catheters are liable to thrombosis; stenosis, and occlusions, so alternative routes are considered as life-saving ways in such cases. Transhepatic permcath is one of the new and up to date methods for inferior vena cava and right atrium catheterization. Few studies with rather limited nomber are available to evaluate transhepatic permcath. The aim of our study is to emphasize the technique, complications, and efficacy of application of percutaneous transhepatic hemodialysis catheters.

Results: Two hundred-ninety six chronic renal dialysis patients were included in this prospective interventional study. They include 180 males and 116 females with mean age of 53.2 years \pm 11.7 years ranging from 38 to 65 years. Percutaneous transhepatic hemodialysis catheters were inserted for all patients. Technical success of the procedure was achieved in all cases (100\%). Post-procedure patency and function of the catheters were followed up with mean follow-up period 750 days. Mean of primary and secondary devices service intervals were 290 and 270 days respectively. Mean time catheter in situ was 280 days. Mean cumulative duration of catheter in situ was 557 days. Catheters migration, sepsis, thrombosis, and exit site infection rates were $0.14,0.15,0.18$, and 0.32 per 100 catheter-days respectively. Three patients had hepatic subcapsular hematoma (1\%). No mortality or other complications were related to the procedure.

Conclusions: Combined ultrasound and fluoroscopic-guided transhepatic permanent dialysis catheter application for patients with exhausted classic venous access routes and non-functioning/thrombosed AV fistulas or grafts showed excellent technical success with good short and mid-term patency rates and low complications rates. Thus, this study encourages us to expand this promising technique for application of dialysis catheter in indicated cases.
\end{abstract}

Keywords: Per-cutaneous transhepatic hemodialysis catheters, Technique, Complications

\footnotetext{
* Correspondence: ooabuelnaga@hotmail.com

'Department of Radio-diagnosis, Faculty of Medicine, Ain Shams University,

Cairo, Egypt

Full list of author information is available at the end of the article
} 


\section{Background}

Chronic kidney disease stage 5 (CKD5) or previously called end-stage renal disease (ESRD) or chronic renal failure is considered as one of the main health problems in Egypt nowadays. The annual incidence in Egypt is estimated to be around 74 per million with total prevalence 264 per million [1].

Arteriovenous (AV) fistulas and grafts are the first line of treatment in such cases. Yet, many patients may sometimes require a catheter-based access for dialysis for various reasons, including thrombosis of those fistulas or grafts, surgical ineligibility, maturation failure, or a need for an urgent dialysis. Center venous dialysis catheters may be short-term non tunneled catheters used for short-term dialysis and should be removed within 7 days or long-term tunneled catheters [2].

The conventional sites for placement of the dialysis catheters are internal jugular, subclavian, and femoral veins. In patients with ESRD, multiple interventions and long-term catheterizations at such conventional sites lead to thrombosis, stenosis, and occlusions of the veins, so alternative routes are considered as life-saving ways in such cases [3].

Transhepatic permcath is one of the new and up-todate methods for inferior vena cava (IVC) and right atrium catheterization. It was first described by Po et al. in 1994 [4] in one case then was further scrutinized in three retrospective studies comprising $12,16,22$, and 38 adult patients in the following years [3,5-7]. So there is only limited experience in using this method with small number of patients involved in different studies.

The aim of our study is to emphasize the technique, complications, and efficacy of application of percutaneous transhepatic hemodialysis catheters in chronic hemodialysis patients with thrombosed conventional sites.

\section{Methods}

This study was approved by the institutional review board. Between January 2015 and October 2018, 296 patients (180 males and 116 females with mean age of 53.2 years \pm 11.7 years ranging from 38 to 65 years) were presented to interventional radiology unit at our university hospital for transhepatic hemodialysis catheter insertion. Causes of the end-stage renal disease were hypertension in 99 patients, diabetes mellitus in 50 patients, renal stones in 28 patients, urinary tract infection in 29 patients, congenital abnormality in 18 patients, primary glomerulonephritis in 15 patients, and unknown causes in 57 patients. All cases were chronic dialysis patients who have thrombosed classic routes of catheterization (internal jugular, subclavian, and femoral veins bilaterally) as well as non-functioning/thrombosed AV fistulas or grafts.
Patients with uncorrectable coagulopathy (platelets count less than 70,000 or INR more than 1.5), patients on long-term anticoagulants, significant abdominal ascites, concurrent active infection, and cirrhotic liver disease patients were excluded from our study.

All patients were subjected to history taking and duplex examinations to verify thrombosis of conventional sites for hemodialysis catheters, abdominal sonography, and duplex to exclude ascites; delineate, and assess both hepatic parenchyma as well as hepatic veins. Lastly, laboratory tests including platelets count and coagulation profile were performed.

The procedure was simply explained to the patients. Then a written consent was obtained from all patients included in the study.

Interventional radiology specialist with 10-year experience in interventional radiology performed the procedures. Another interventional radiology specialist with 5-years experience assisted him.

All procedures were performed in interventional radiology unit at university hospital.

\section{Technique}

Single dose of broad spectrum intra-venous antibiotic (Cefazolin $1 \mathrm{~g}$ ) was administrated $2 \mathrm{~h}$ before the procedure. Patients lie in supine position, and the right side of the skin of abdomen and lower chest was sterilized with Betadine $^{\circ}$ Solution (Povidone-iodine, 10\%). All patients were monitored for their vital signs including pulse, blood pressure, and oxygen saturation during the whole time of procedure. The procedure was done under local anesthesia with injection of $10 \mathrm{ml}$ lidocaine. Some patients were given mild sedation (midazolam $0.03 \mathrm{mg} / \mathrm{kg}$ intravenous) to alleviate their anxiety. The chosen hepatic veins, right in 128 patients (Figs. 1 and 2), middle in 110 patients (Figs. 3 and 4), and left in 58 patients (Fig. 5) were accessed by a 16-G angiocath under ultrasound guidance. We used either intercostal or subcostal approaches for right and middle hepatic veins and subxyphoid approach for left hepatic vein. We aim to enter the hepatic veins rather than IVC directly to allow a longer intra-vascular tract thus reducing the possibility of migration out of the vessels. Entrance of the hepatic veins is confirmed by injection of diluted contrast media (Ultravist 300 (iopromide) till become visualized. Then under fluoroscopic guidance (TOSHIBA, Infx-8000v), a hydrophilic guide wire $(0.035 \mathrm{in}$.) was introduced through the angiocath in hepatic vein, and secured upwards in superior vena cava (SVC) and right atrium in 234 patients or downwards in inferior vena cava in 60 patients, a split catheter was applied in 2 cases where one end of the catheter was within the right atrium and the other end within the IVC (Fig. 6). Subcutaneous tunnel was formed anteroinferiorly and then the catheter 


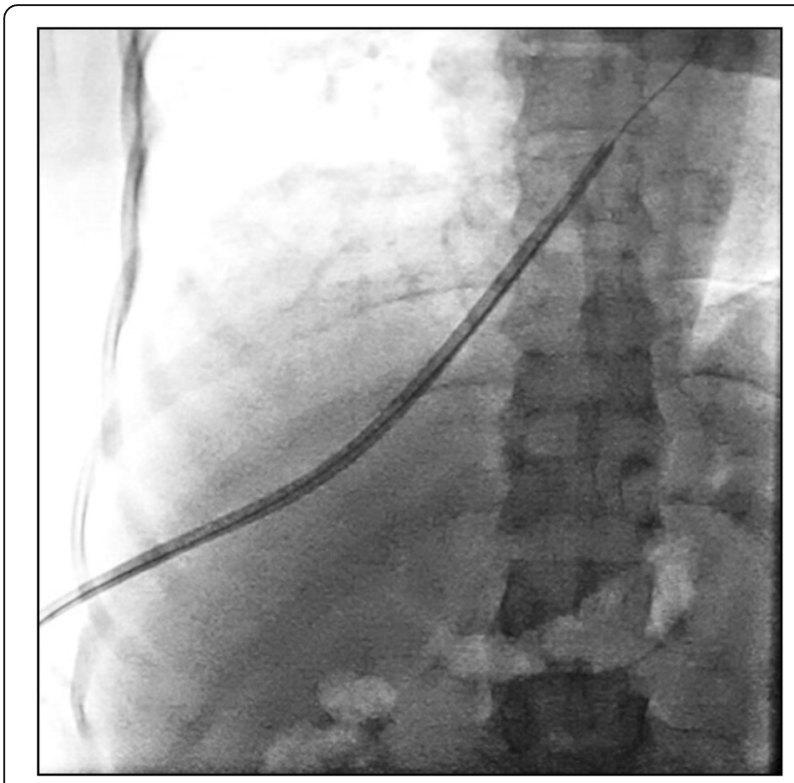

Fig. 1 Catheter introduced through right hepatic vein up to the atrium. X-ray upper abdomen frontal projection showing

transhepatic hemodialysis catheter introduced through right hepatic vein with catheter tip within the right atrium

was inserted over the wire through the peel away sheath. Catheters used in our study were Hickman hemodialysis/ apheresis $13.5-\mathrm{F}$., round dual-lumen radiopaque silicone catheter, luer lock adapters, and SureCuff tissue in growth cuff, $2.0 \mathrm{~mm}$ in lumens, $36 \mathrm{~cm}$ in overall length, $19 \mathrm{~cm}$ in tip-to-cuff length (Bard Acess System, Salt Lake City,

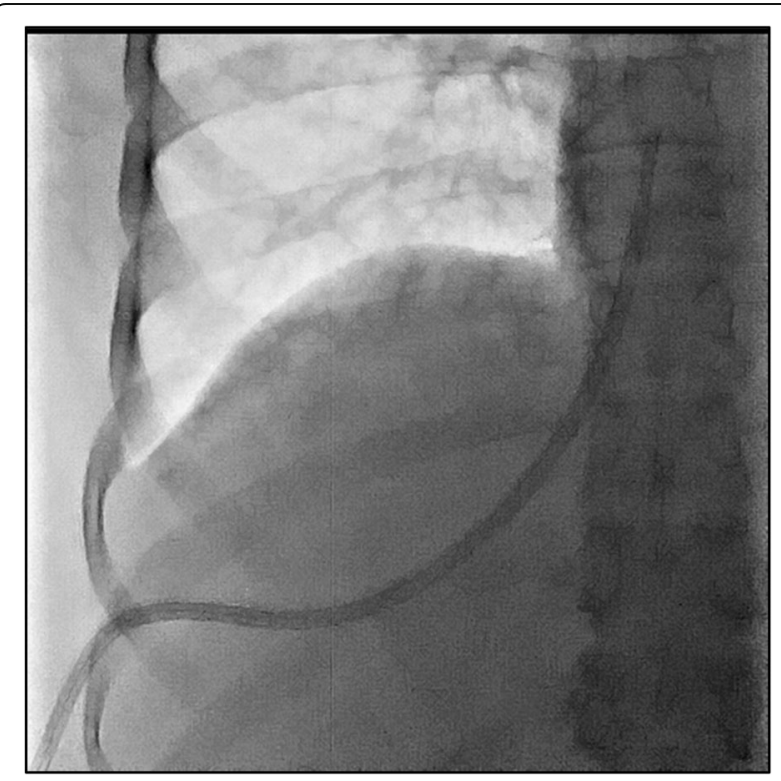

Fig. 2 Catheter introduced through right hepatic vein up to the atrium. X-ray upper abdomen frontal projection showing transhepatic hemodialysis catheter introduced through right hepatic vein with catheter tip within the right atrium

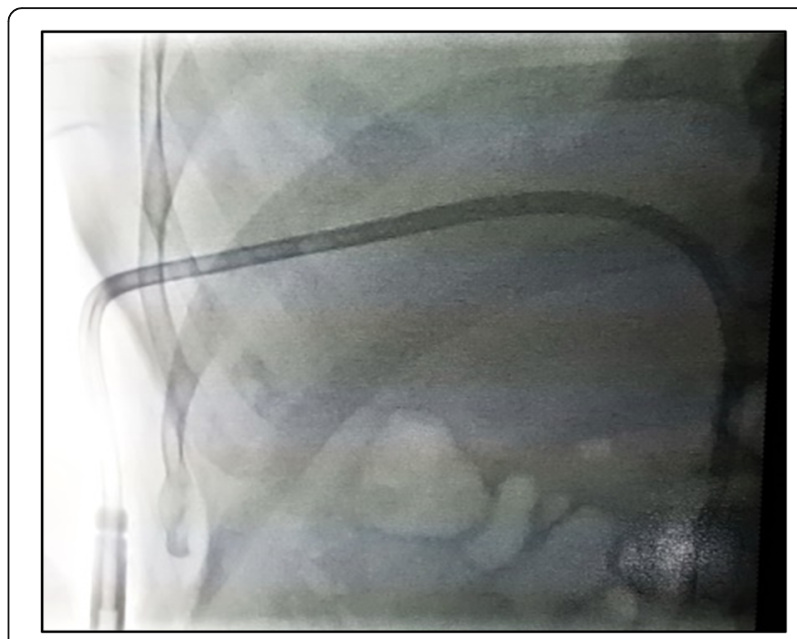

Fig. 3 Catheter introduced through middle hepatic vein down to IVC. X-ray upper abdomen frontal projection showing transhepatic hemodialysis catheter introduced through middle hepatic vein with catheter tip within IVC

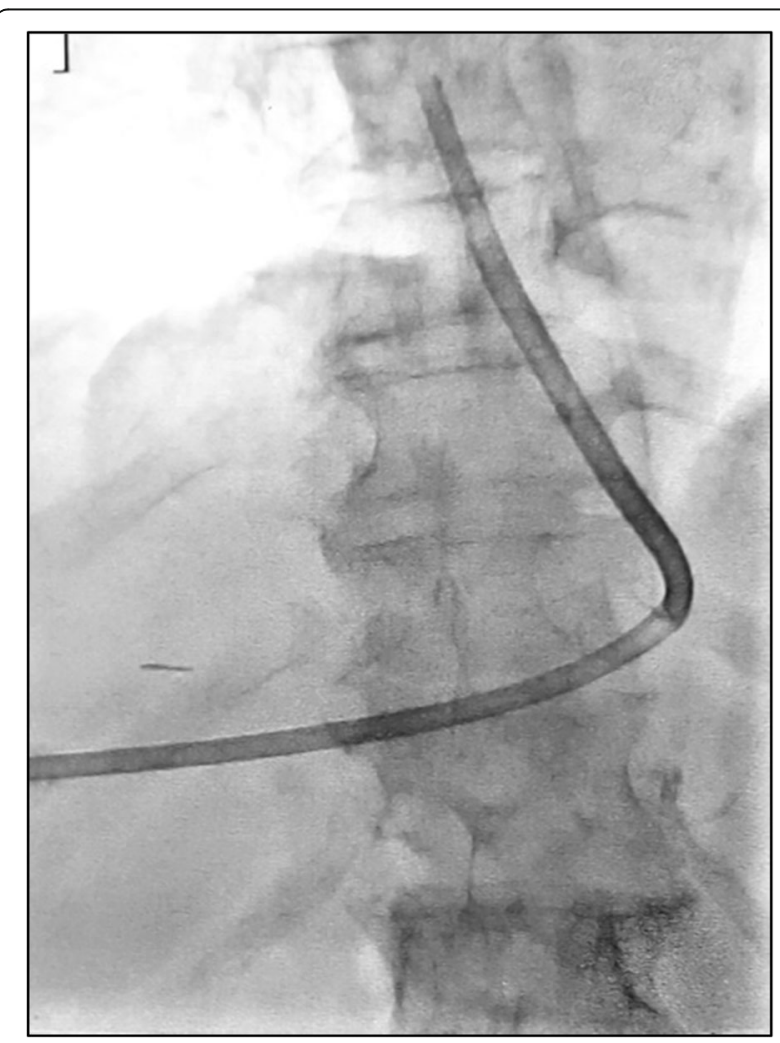

Fig. 4 Catheter introduced through middle hepatic vein up to the atrium. X-ray upper abdomen frontal projection showing transhepatic hemodialysis catheter introduced through middle hepatic vein with catheter tip within the right atrium 


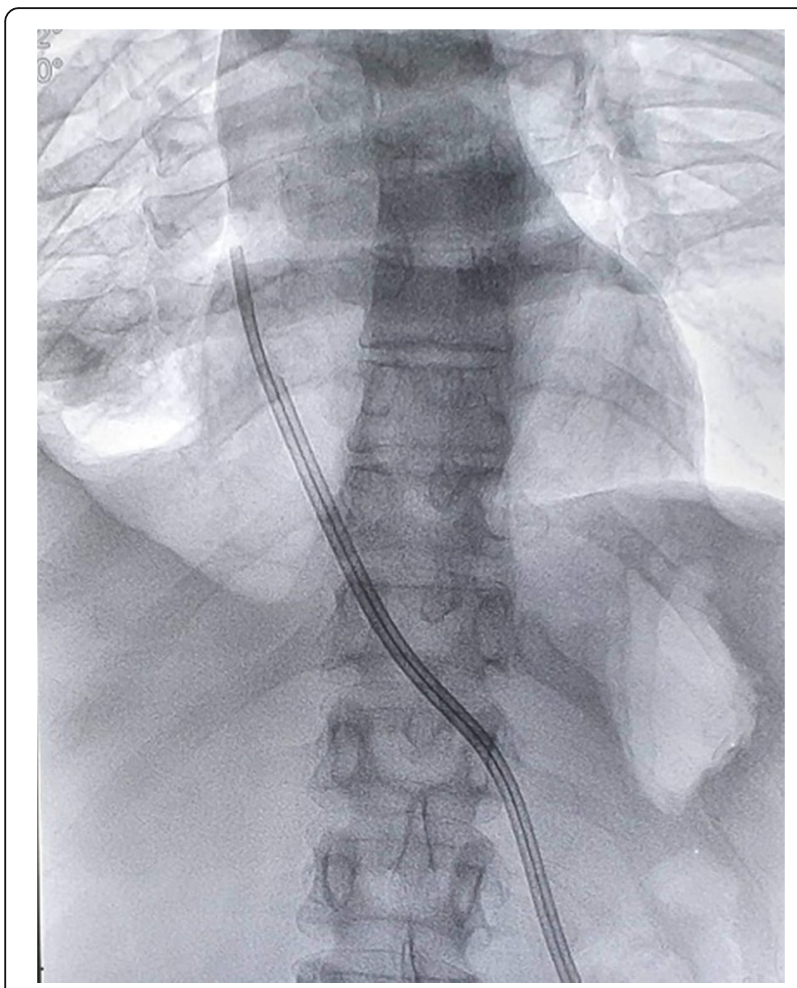

Fig. 5 Catheter introduced through left hepatic vein up to the atrium. X-ray upper abdomen frontal projection showing transhepatic hemodialysis catheter introduced through left hepatic vein with catheter tip within the right atrium

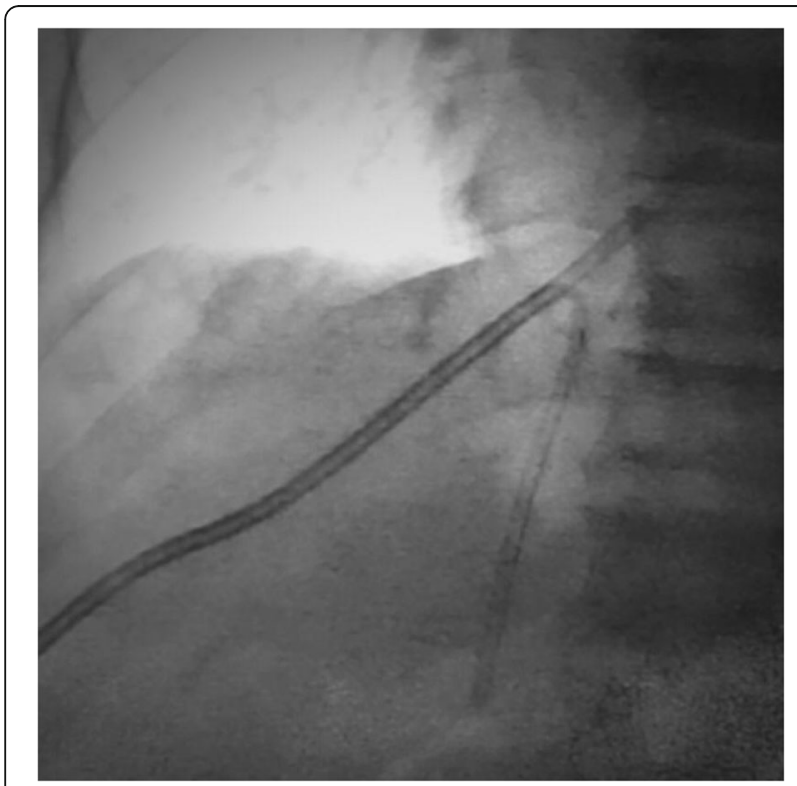

Fig. 6 Split catheter introduced through right hepatic vein up to the atrium and down to IVC. X-ray upper abdomen frontal projection showing transhepatic hemodialysis split catheter introduced through right hepatic vein with catheter tips, one within the right atrium and the other within IVC
Utah), or Ash Split Cath (Medcomp, Harleyville, Pa) in different lengths. The catheter tip was positioned opposite the third intercostal space to be within the right atrium. In other cases that have catheter tip within IVC, it was positioned opposite or above the level of L1 vertebra. The venotomy site was closed using silk sutures $(2 / 0)$. Both lumens of the catheter were flushed with heparin and aspirated to ensure patency. Post procedural images were obtained to confirm the correct positioning. The dialysis catheter can be used directly after its insertion.

The average procedure time was $55 \mathrm{~min}$ ranged between 40 and $100 \mathrm{~min}$.

\section{Post-procedure care}

Pain killers were prescribed and taken on demand. Most of patients respond to oral analgesic (non steroidal antiinflammatory drugs). We have to give stronger intravenous analgesics (pelfergan and tramal) in few cases. All cases were admitted at the same day of the procedure. Cases without complication were routinely discharged $24 \mathrm{~h}$ after the procedure. Sonographic follow-up 24-h post-procedure was performed before discharge to exclude any complications specially hepatic subcapsular hematoma and intra-peritoneal bleeding.

Follow-up of catheter for its patency and function was done via documentation by the nephrologists at dialysis centers and communication with the interventional radiology specialist. Follow-up was performed by monitoring the catheter dialysis rate in each session. Also, inspection of catheter exit was done to exclude infection. Blood cultures obtained from the catheter site was done if catheter-related sepsis is suspected. All complications were monitored and documented. Catheters which are no longer functioning or cases with suspected catheterrelated complications were sent again to the interventional radiology unit for reassessment and revision if indicated.

Catheter exchange through same primary access site was indicated and performed 315 times in seventy-two patients. Causes of catheter exchange were catheter thrombosis, sepsis, and migration.

\section{Definitions}

All the terms used in the study were defined in light of the "Reporting Standards for Central Venous Access" definitions of Society of Interventional Radiology (SIR) as follows: Technical success is defined as introduction into the venous system with the tip positioned in the preferred location and with adequate catheter function $(300 \mathrm{~mL} / \mathrm{min})$. Primary device service interval is defined as the number of catheter-days from initial placement until removal. Secondary device service interval is defined as the number of catheter-days after device replacement using the same access site. Mean time 
catheter in situ is defined as the cumulative catheterdays divided by the cumulative number of catheters in the entire study population. Mean cumulative duration of catheter in situ is defined as the cummulative catheter-days divided by the number of patients.

Mean duration of function of catheters, mean of primary device service (function), mean of secondary device service (function) interval, mean time catheter in situ and mean cumulative duration of catheter in situ were calculated using Kaplan-Meier method.

\section{Results}

Two hundred ninety-six chronic renal dialysis patients were included in our study. They include 180 males (61\%) and 116 females (39\%). Mean age of the patients was 53.2 years \pm 11.7 years ranging from 38 to 65 years (Table 1).

Hepatic veins accessed as well as final position of the hemodialysis catheter are described in Table 2.

Technical success was achieved in all cases (100\%).

Exchange of catheter was indicated and done 315 times in seventy-two patients with average number of catheters used for each patient was 4.5.

Mean duration of function of catheters is summarized in Table 3.

Mean durations of function of catheters are detailed as follows:

Mean of primary device service (function) interval was 290 days. Mean of secondary device service (function) interval was 270 days. Mean time catheter in situ was 280 days. Mean cumulative duration of catheter in situ was 577 days.

Mortality and complications of the procedure and catheters are summarized in Table 4.

Mortality and complications of the procedure and catheters are detailed as follows:

No mortality was reported related to catheter application. Three cases developed small subcapsular hematomas ranging in size between 1 and $2.5 \mathrm{~cm}$ at their maximum dimensions. These hematomas resolved spontaneously on serial sonographic follow-up and did not necessitate specific management. Catheter migration rate

Table 1 Etiology of end-stage renal disease

\begin{tabular}{lll}
\hline Etiology & Number & Percentage \\
\hline Hypertension & 99 & $33 \%$ \\
Diabetes mellitus & 50 & $17 \%$ \\
Renal stones & 28 & $10 \%$ \\
Urinary tract infection & 29 & $10 \%$ \\
Congenital abnormality & 18 & $6 \%$ \\
Primary glomerulonephritis & 15 & $5 \%$ \\
Unknown causes & 57 & $19 \%$ \\
\hline
\end{tabular}

Table 2 Hepatic veins access and final position of the catheter

\begin{tabular}{|c|c|c|c|c|c|c|c|}
\hline \multirow[t]{2}{*}{ Cases } & \multicolumn{4}{|c|}{ Hepatic veins access } & \multicolumn{3}{|c|}{ Position of the catheter } \\
\hline & & Right & Middle & Left & Right Atrium & IVC & Both \\
\hline & Number & 128 & 110 & 58 & 234 & 60 & 2 \\
\hline & Percentage & $43 \%$ & $37 \%$ & $20 \%$ & $79 \%$ & $20 \%$ & $1 \%$ \\
\hline
\end{tabular}

was 0.14 per 100 catheter-days. The catheters withdraw back into the hepatic vein and thus no more functioning. This was managed by repositioning with or without catheter exchange. Sepsis rate was 0.15 per 100 catheterdays. Diagnosis of sepsis depended upon clinical symptoms including fever and chills in catheter-dependent patients with no localizing signs. This was followed by laboratory confirmation through positive blood cultures obtained from the catheter. This was managed with catheter replacement and antimicrobial therapy. Catheter thrombosis rate was 0.18 per 100 catheter-days. This was managed with catheter replacement. Exit site infection (including skin erythema, induration, and wound discharge) rate was 0.32 per 100 catheter-days. This did not neccessitate catheter exchange. It was managed with local anti-microbial and sterile pads. Other complications including intra-peritoneal hemorrhage, contrast reaction, and intra-venous thrombosis were not encountered in our study. Post-procedure mild transient right hypochondrial pain which was controlled by pain killers for short duration was considered as frequent post-procedure consequences rather than a complication.

There were no statistically significant differences in catheter longevity or complications between different hepatic veins approaches.

\section{Discussion}

Nowadays, transhepatic venous route is considered to be safe and functional in patients with exhausted all other central venous accesses when performed using different imaging modalities and by experienced hands. Transhepatic catheterization was first described by Po et al. in 1994 [4] on an adult patient with end-stage lupus nephritis who had no alternative for vascular access and failed peritoneal dialysis; it was technically successful with no bleeding or thrombotic complications, and the patient underwent dialysis from that vascular access for a year [4]. This study was followed by other studies done on

Table 3 Mean durations of function of catheters

\begin{tabular}{lc}
\hline & Days \\
\hline Mean of primary device service interval & 290 \\
Mean of secondary device service interval & 270 \\
Mean time catheter in situ & 280 \\
Mean cumulative duration of catheter in situ & 577 \\
\hline
\end{tabular}


Table 4 Mortality and complications rate

\begin{tabular}{ll}
\hline & Rate \\
\hline Procedure-related mortality & $0 \%$ \\
Hepatic subcapsular hematoma & $3 / 296(1 \%)$ \\
Catheter migration & 0.14 per 100 catheter-days \\
Sepsis & 0.15 per 100 catheter-days \\
Catheter thrombosis & 0.18 per 100 catheter-days \\
Exit site infection & 0.32 per 100 catheter-days \\
Intra-peritoneal hemorrhage & $0 \%$ \\
Contrast reaction & $0 \%$ \\
Intra-venous thrombosis & $0 \%$ \\
\hline
\end{tabular}

limited number of patients with limited data on the long-term effectiveness [3-7].

So we performed our study on 296 patients who had thrombosed conventional vascular accesses as well as non-functioning or thrombosed AV fistulas or grafts.

We used in our study transhepatic approach rather than the translumbar approach which is another alternative vascular access for patients with inaccessible vascular route. Some studies argue that the transhepatic approach is superior over the translumbar one; this can be attributed to many causes. For examples, transhepatic approach can be used in cases with occluded IVC. It has lower risk of hemorrhage and migration. And in case of hemorrhage, there is a chance of transhepatic or endovascular embolization. Beside that, translumbar catheter revisions are more difficult because of a risk of retroperitoneal fibrosis [5, 8-11].

In comparison to previous study done by El Garib et al. [12], our study has larger population as well as longer follow-up period. Our study population was much larger 296 patients versus 23 patients in his study. Our follow-up period was 750 days versus 365 days in his study. Our technical success rate was $100 \%$ while his technical success rate was $88 \%$. Mean cumulative duration of catheter in situ in our study was 577 days while in his study, maximum duration of catheter in situ was 300 days.

We had technical success in all patients (100\%). The same technical success was reported by Joaquim et al. [13]. In our study, the most common hepatic vein access was the right hepatic vein followed by middle hepatic vein and lastly left hepatic vein. We prefer the right hepatic vein as a first choice as it is peripheral (near the puncture site) as well as it obtains a horizontal upper part towards the IVC. Joaquim et al. [13] uses the right hepatic vein in all cases, also El Garib et al. [12] uses either right or middle hepatic veins. In our study, the tip of the hemodialysis catheter was in the right atrium in most cases ( $80 \%$ of cases), and the IVC in $20 \%$ of cases. This is coinciding with El Garib et al. [12] whose catheter tip was put in the right atrium in $80 \%$ of cases, IVC in $16 \%$ of cases, and SVC in $4 \%$ of cases. Joaquim et al. [13] put the catheter tip at the junction between SVC and right atrium in all six cases he performed.

In our study, the mean of functioning catheter was 280 days. This duration is higher than Biswal et al. [11] who obtain a mean of 250 days for hemodialysis catheter inserted via translumbar approach. Yet it is less than Joaquim et al. [13] who obtain a mean of 300.5 days functioning catheter in his small-sized study (only six patients).

We agree with El Garib et al. [12] that exit site infection is the most common complication encountered in both studies. This complication did not necessitate catheter exchange. In our study, the most common complication that led to catheter dysfunction is catheter thrombosis (0.18 per 100 catheter-days). This rate is matching with Younes et al. [7] and lower than the rate of Stavropoulos et al. [5], Smith et al. [6] and Sanal et al. [3]. Stavropoulos et al. [5]. This is may be attributed to washing the catheter with heparinized saline after each procedure. These precautions reduce the incidence of thrombosis significantly. The third most common complication in our study was infection with rate of 0.15 per 100 catheter-days. This rate is slightly higher than El Garib et al. [12] who had infection rate 0.13 per 100 catheter-days yet much lower than Stavropoulos et al. [5] and Younes et al. [7] who both had sepsis rate 0.22 per 100 catheter-days. The fourth most common complication in our study was migration with rate of 0.14 per 100 catheter-days. This rate is much lower than Younes et al. [7] rate which was 0.39 per 100 catheterdays. This might be due to that we did our best to access the hepatic veins as peripheral as we can which permits greater intra-vascular tract and thus more stability and less liability for migration and dislodgement of the catheters. We had three cases that developed hepatic subcapsular hematoma. The three cases had successful conservative management. No procedure or catheterrelated mortality occurred in our or other studies. Also we had no cases with intra-peritoneal hemorrhage or contrast reaction which emphasizes safety of the procedure.

In our study, exchange or removal of catheter did not result in bleeding, and path emolization was not indicated and not performed. On the contrary, Joaquim et al. [13] performed gel foam embolization for the catheter path in all his six patients to prevent bleeding.

This rather large population study emphasizes safety of procedure. With more practice and better knowledge, lower complication rates and longer patency and functioning duration of the transhepatic hemodialysis catheters will be achieved.

The limitation of this study is lack of longer follow-up for catheters patency and function. 


\section{Conclusions}

Combined ultrasound and fluoroscopic-guided transhepatic permanent dialysis catheter application for patients with exhausted classic venous access routes and nonfunctioning/thrombosed AV fistulas or grafts showed excellent technical success with good short and mid-term patency rates and low complications rates. Thus, this study encourages us to expand this promising technique for application of dialysis catheter in indicated cases.

\section{Abbreviations}

AV: Arteriovenous; CKD5: Chronic kidney disease stage 5; ESRD: End-stage renal disease; IVC: Inferior vena cava; SIR: Society of Interventional Radiology; SVC: Superior vena cava

\section{Acknowledgements}

We would like to acknowledge the hospital administrators who save no effort to provide us with the materials needed in our study.

\section{Authors' contributions}

KA performed the procedure with pre-procedural assessment of patients as well as following up patients and managing the complications. OA designed the study and performed statistical analysis and assisted to draft the manuscript. HI compared our work to other similar studies and drafted the manuscript. GS assisted in performing the procedure and assessment of patients before the procedure and managing the complications. RS followed up patients for patency and functioning of the hemodialysis catheters as well as diagnosing complications. All authors read and approved the manuscript.

\section{Funding}

No funding was obtained for this study.

\section{Availability of data and materials}

The datasets generated and analyzed during the current study are available from the corresponding author on reasonable request.

\section{Ethics approval and consent to participate}

It was taken from our institutional ethical committee/Radiology department, Ain Shams University, with informed written consent taken from each patient. The reference number is not applicable as it was a departmental approval to use the patients' data and material with no need to change patients' management strategy as we used well established technique and management strategy in our institution.

\section{Consent for publication}

All patients included in this research gave written informed consent to publish the data contained within this study. If the patient was deceased or unconscious when consent for publication was requested, written informed consent for the publication of this data was given by their parent or legal guardian.

\section{Competing interests}

The authors declare that they have no competing interests.

\section{Author details}

${ }^{1}$ Department of Radio-diagnosis, Faculty of Medicine, Ain Shams University, Cairo, Egypt. ${ }^{2}$ Internal Medicine Department, Faculty of Medicine, Ain Shams University, Cairo, Egypt.

Received: 25 July 2019 Accepted: 29 October 2019

Published online: 12 December 2019

\section{References}

1. Ahmed AM, Allam MF, Habil ES, Metwally AM, Ibrahiem NA, Radwan M, Gadallah MA (2010) Development of practice guidelines for hemodialysis in Egypt. Ind J Nephrol 20(4):193-202

2. Rahman S, Kuban JD (2017) Dialysis catheter placement in patients with exhausted access. Tech Vasc Interv Radiol. 20(1):65-74
3. Şanal B, Nas ÖF, Doğan N et al (2016) Safety and functionality of transhepatic hemodialysis catheters in chronic hemodialysis patients. Diagn Interv Radiol. 22(6):560-565

4. Po CL, Koolpe HA, Allen S, Alvez LD, Raja RM (1994) Transhepatic perm cath for hemodialysis. Am J Kidney Dis 24:590-591

5. Stavropoulos SW, Pan JJ, Clark TW et al (2003) Percutaneous transhepatic venous access for hemodialysis. J Vasc Interv Radiol 14:1187-1190

6. Smith TP, Ryan JM, Reddan DN (2004) Transhepatic catheter access for hemodialysis. Radiology 232:246-251

7. Younes HK, Pettigrew CD, Anaya-Ayala JE et al (2011) Transhepatic hemodialysis catheters: functional outcome and comparison between early and late failure. J Vasc Interv Radiol 22:183-191

8. Silberzweig JE, Sacks D, Khorsandi AS, Bakal CW (2003) Reporting standards for central venous access. J Vasc Interv Radiol. 14:443-452

9. Lund GB, Lieberman RP, Haire WD, Martin VA, Kessinger A, Armitage JO (1990) Trans-lumbar inferior vena cava catheters for long-term venous access. Radiology. 174:31-35

10. Rajan DK, Croteau DL, Sturza SG, Harvill ML, Mehall CJ (1998) Translumbar placement of inferior vena caval catheters: a solution for challenging hemodialysis access. Radiographics. 18:155-167

11. Biswal R, Nosher JL, Siegel RL, Bodner $L$ (2000) Translumbar placement of paired hemodialysis catheters (Tesio catheters) and follow-up in 10 patients. Cardiovasc Intervent Radiol. 23:75-78

12. El Gharib M, Niazi G, Makkeyah WHY (2014) Transhepatic venous catheters for hemodialysis. Egypt J Radiol Nucl Med 45(2):431-438

13. da Motta-Leal-Filho JM, Carnevale FC, Nasser F, de Oliveira Sousa Junior W, Zurstrassen CE, Moreira AM, Affonso BB, Cerri GG (2010) Percutaneous transhepatic venous access for hemodialysis: an alternative route for patients with end-stage renal failure. J Vasc Bras 9(3):131

\section{Publisher's Note}

Springer Nature remains neutral with regard to jurisdictional claims in published maps and institutional affiliations.

\section{Submit your manuscript to a SpringerOpen ${ }^{\circ}$ journal and benefit from:}

- Convenient online submission

- Rigorous peer review

- Open access: articles freely available online

High visibility within the field

- Retaining the copyright to your article

Submit your next manuscript at $\boldsymbol{\nabla}$ springeropen.com 